\title{
Planificación urbana estratégica a través de macroproyectos urbanos: percepción ciudadana sobre el parque lineal "La Ronda del Sinú" en la ciudad de Montería, Colombia
}

\author{
Strategic urban planning through urban macro projects: citizen perception of the linear park "La Ronda del Sinú" in the city \\ of Montería, Colombia
}

Rosa Babilonia *, Rafael Sánchez *, Juan Jiménez Caldera** y Gren Durango**

Recibido: 23 de marzo de 2018

Aceptado: 29 de noviembre de 2018

\begin{abstract}
Resumen
Desde inicios de siglo, la planificación urbana estratégica ha predominado en el modo de organizar, concebir e imaginar a las ciudades latinoamericanas. Siguiendo sus preceptos, los centros urbanos comenzaron a elaborar estrategias que les permitieran lidiar y posicionarse a nivel mundial, siendo los proyectos urbanos íconos una herramienta muy eficaz, pues les permitirán lograr una rápida singularidad y notoriedad en el marketing urbano internacional y con ello, la capacidad de canalizar y atraer nuevos flujos de inversión. La ciudad colombiana de Montería, buscará mediante el parque lineal Ronda del Sinú modificar la imagen de una ciudad intermedia caracterizada por las desigualdades socioeconómicas provocadas por la violencia y el desempleo. A una década del inicio de este proceso es buen momento para evaluar los resultados del proyecto implementado. A través de entrevistas a los habitantes/usuarios fue posible identificar que si bien se reconoce que estéticamente la orilla del río Sinú ha mejorado y ahora Montería es conocida nacional e internacionalmente, se identifican una serie de deficiencias y problemáticas que emergieron a raíz de la construcción del proyecto. El Parque Lineal Ronda del Sinú quiso ser un ejemplo de planeamiento estratégico urbano, pero incorporó una escasa capacidad de diálogo, centrándose en la expresión de interés material, más que en las necesidades reales de los habitantes, surgiendo desequilibrios entre lo proyectado en la etapa de diseño del plan estratégico y los resultados obtenidos después de su materialización.
\end{abstract}

Palabras clave: espacio público, habitantes, planificación urbana estratégica, proyectos urbanos.

\begin{abstract}
Since the beginning of the century, strategic urban planning has dominated the organization, conception and imagination of Latin American cities. Following its precepts, urban centers began to develop strategies that would allow them to deal with and position themselves at the global level. Urban icons are a tool that allow cities to rapidly achieve singularity and notoriety in international urban marketing and with this the capacity to attract and bring new influx of investment. The Colombian city of Montería, expected the creation of the lineal park Ronda del Sinú to change their image. Prior to this project, the city surrounding it was characterized by socioeconomic inequality which led to violence and unemployment. 10 years after the inauguration of the project, one can evaluate the results of it. After interviewing people who lived in the area, it was possible to identify that the aesthetic of the area surrounding the river had improved, which has given Montería national and international notoriety. However, it is also apparent that there are a series of deficiencies and problems that emerged as a result of the actualized project. The Lineal Park, Ronda de Sinú, could be an example of strategic urban planning, but due to the lack of dialogue with current residents, it was centered in material interests more than actual needs of the residents. This led to discrepancies between the final results obtained and the initial plan during the design stage.

Keywords: inhabitants, public space, strategic urban planning, urban projects.
\end{abstract}

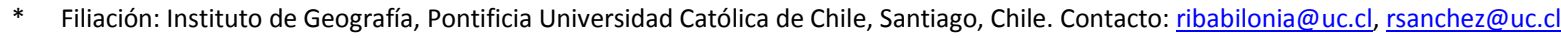

** Filiación: Departamento de Geografía, Universidad de Córdoba, Montería, Colombia. Contacto: juanjimenezc@correo.unicordoba.edu.co, johanadurango@hotmail.com

Cómo citar: Babilonia, R., Sánchez, R., Jiménez Caldera, J. y Durango, G. (2018). Planificación urbana estratégica a través de macroproyectos urbanos: percepción ciudadana sobre el parque lineal "La Ronda del Sinú" en la ciudad de Montería, Colombia. Revista de Urbanismo, 39, 1-12. https://doi.org/10.5354/0717-5051.2018.48727 


\section{Introducción}

En los últimos años, los proyectos urbanos se han convertido en una de las estrategias más utilizadas por las autoridades gubernamentales y los actores privados para impulsar la inversión y reconversión de las ciudades colombianas. Montería, capital del departamento de Córdoba en la región del Caribe colombiano, experimentó a partir de mediados de la década de 1980 una etapa de detrimento estimulado por un conflicto armado complejo que generó un estancamiento económico, un incremento de los asentamientos ilegales y un rápido deterioro de su equipamiento e infraestructura. Frente a este escenario, el gobierno local buscó superar esta realidad mediante, entre otros, la generación de un ambicioso y cuestionado proyecto de transformación denominado macroproyecto urbano "La Ronda del Sinú".

Como consecuencia del abandono de la teoría económica keynesiana, la planificación urbana en América Latina adopta nuevos enfoques que buscan responder a la complejidad, diversidad y problemáticas que la urbanización global impone a los centros urbanos (De Mattos, 2010). El nuevo modelo económico propuesto, con un Estado centrado en la regulación y unos actores privados fortalecidos, empujó a adoptar un planeamiento urbano basado en la descentralización, la gobernanza y el aprovechamiento de las ventajas comparativas y competitivas de los territorios. Así la planificación estratégica, que proviene del mundo empresarial, es aplicada a los centros urbanos con el objetivo de incentivar la competencia por las inversiones, construir estrategias de marketing, creando una imagen o marca identitaria (Duque, 2013).

Bajo este paradigma, varias ciudades latinoamericanas inician una serie de proyectos iconos que las posicionará en el sistema urbano global. Aprovechando el ciclo positivo en el precio de las materias primas, de la primera década del siglo XXI, se construyeron parques y áreas verdes, se restauran microcuencas urbanas, se mejoró el mantenimiento y prestación eficiente de los servicios públicos domiciliarios, se privilegió el desarrollo de sistemas de transportes masivos, se extendieron las autopistas urbanas, se renovaron los centros históricos, se construyeron grandes monumentos y edificios, entre otros elementos (Brahm, 2005; Figueroa, 2005; Martínez, 2003).

En el caso colombiano, con la publicación de las políticas de "Ciudades y Ciudadanías de 1995" y la Ley 388 de 1997, que derogó la Reforma Urbana de 1989, y otros cuerpos legales posteriores, se estableció un enfoque de ordenamiento orientado a otorgar un mayor protagonismo de los gobiernos locales en el planeamiento estratégico, gestión y supervisión de sus territorios (Duque, 2011). De esta manera, gran parte de las estrategias de intervención urbanística implementadas en las ciudades colombianas han sido promovidas por los gobiernos locales y han implicado identificar áreas de renovación y transformación para estimular el establecimiento de actividades económicas como el comercio y el turismo.

Si bien, como señala Steinberg (2001) el éxito de la planificación urbana depende de la voluntad política de los alcaldes y las autoridades locales, de la constelación institucional de actores claves y de los enfoques temáticos; un elemento clave será el valor y evaluación que los ciudadanos posean con respecto a la satisfacción de sus necesidades al momento proyectar y construir la ciudad (Roitman, 2008). Precisamente, este último aspecto es el que se indaga en este artículo sobre el proyecto urbano "La Ronda del Sinú" de la ciudad de Montería.

El objetivo es contrastar el proceso de planificación urbana estratégica llevada a cabo por el gobierno local de la ciudad, con las diferentes percepciones y valoraciones que los habitantes/usuarios monterianos poseen sobre el parque lineal Ronda del Sinú. Desde el punto de vista metodológico, el trabajo utiliza información cualitativa primaria obtenida a través de entrevistas semiestructuradas aplicadas a ciudadanos y comerciantes que se encontraron en el área de influencia directa del proyecto ${ }^{1}$.

El texto se estructura en tres partes. En la primera se contextualiza el surgimiento de la planificación urbana estratégica como catalizador de la globalización actual y como un instrumento que permite identificar las

\footnotetext{
${ }^{1}$ Se identificó como área de influencia del parque el espacio ubicado entre las calles 29 y 35 con carreras 1 y 4 del barrio El Centro.
} 
fortalezas y debilidades de las ciudades. Posteriormente, se describe el marco legislativo que permitió el arribo de los proyectos urbanos estratégicos en los asentamientos colombianos desde fines de la década de 1990. Finalmente, se realiza un análisis de la percepción que los habitantes/usuarios poseen del proyecto urbano "La Ronda del Sinú" en la ciudad de Montería.

\section{Planificación urbana estratégica. La emergencia de los proyectos urbanos}

Hacia finales del siglo XX, la multiplicación de los asentamientos y la agudización de las problemáticas urbanas a nivel mundial volvió a colocar al diseño y la planificación urbana, que habían tenido un periodo de letargo, como una respuesta válida y concreta a los dilemas de los asentamientos urbanos (Abbott, 2006; ElKhoury \& Robbins, 2013; Fainstein, 1999; Mawromatis, 2013).

A partir de la publicación del Informe Brundtland en 1987, la celebración de la Cumbre de la Tierra y la aprobación de la Agenda 21 en 1992, la mayoría de los países establecieron una serie de objetivos encaminados a resolver los problemas de contaminación, deterioro de recursos naturales, pobreza, protección de la atmósfera, conservación de la diversidad biológica, entre otros. En este contexto, en la planificación urbana adquiere mayor interés el concepto de desarrollo sostenible, emergiendo la denominada planificación urbana sistémica o sostenible, la cual trata de incluir la dimensión ambiental desde las fases iniciales del proceso hasta las últimas etapas de su ejecución (Tamayo, 2008).

La planificación urbana sistémica supone la existencia de una estrecha relación entre la ciudad y su entorno, de modo que la urbe no es considerada como un sistema estático o cerrado, sino que la extensión de las ciudades sobre las tierras agrícolas productivas o las áreas naturales de sus periferias, así como la contaminación de aguas y aire no pueden ser ajenas a la gestión de los centros urbanos (Echabarría y Aguado 2003). Rápidamente, el ambientalismo y desarrollo sostenible aparecen como verdades imperturbables, adquiriendo una sacralización de ideología espacial global del desarrollo urbano, que conduciría a la construcción de un modelo orgánico de la estructuración espacial y forma urbana (Gunder, 2010).
Bajo esta perspectiva, la planificación urbana debía contar con una visión a largo plazo, sistémico e integral basado en un plan paisajista, que prevea y minimice los impactos ambientales, que sea capaz de articular todos estos resultados en un plan de usos de suelo y tuviera la suficiente flexibilidad de someterse a un análisis espacial de carácter continuo, así como permitir una planificación orientada a proyectos concertados de desarrollo sostenible (Brand, 1995; Echabarría y Aguado, 2003).

No obstante lo anterior, en los primeros años del siglo XXI, debido a la inestabilidad económica y política que muchos países en vía de desarrollo experimentarán hacia finales de la década de 1990 (e.g. crisis financiera asiática en 1997, Rusia en 1998, Ecuador en 1999, Argentina en 2001) esta perspectiva de planificación sistémica perderá importancia, en desmedro de la creación de proyectos urbanos de infraestructura y equipamiento icónicos que buscarán mejorar las condiciones físicas de las ciudades y posicionarlas como referentes, tanto a nivel nacional como en el diseño urbano mundial (Precedo, Orosa y Míguez, 2010).

La consolidación y profundización de las directrices económicas neoliberales, la aceleración de las innovaciones tecnológicas y la necesidad de acumulación y flujo masivo de capitales globales, consolidará la idea de ver a las ciudades, como una empresa competitiva, que debe proveer las condiciones materiales idóneas para el intercambio financiero, comercial e ideológico; en tanto, la gestión pública deberá asegurar los marcos políticos, legales y tributarios adecuados para asegurar y garantizar la inversión privada (Sierra, 2016). De esta manera, se retoman las ideas del planeamiento estratégico de la década de 1970 en Estados Unidos, vinculado al desarrollo económico local, la promoción de lugares particulares y las alianzas público-privadas (Duque, 2011).

La planificación urbana estratégica emerge para convertir a los centros urbanos en catalizadores de la globalización actual, la cual se caracteriza por una enorme incertidumbre sobre el futuro, ser altamente competitiva y un entorno cada vez más dinámico e incierto (Duque, 2013; Pradilla, 2009). En este escenario, la planificación urbana estratégica sería una herramienta que permite anticiparse a las nuevas tendencias, discontinuidades y sorpresas, y concentrarse en las nuevas oportunidades. Al centrarse en un número limitado de áreas temáticas, logra 
tener visión crítica del entorno en cuanto a la determinación de fortalezas y debilidades en el marco de las oportunidades y amenazas, identificando los principales interesados (públicos y privados) permitiendo una amplia y diversa participación en el proceso de planificación del sector público, la sociedad civil, la sociedad económica y el gobierno (Albrechts, 2004; Steinberg, 2001).

Así, los centros urbanos comenzarán a elaborar estrategias que les permitan lidiar y posicionarse en el sistema urbano global, mediante la exaltación de sus ventajas competitivas y comparativas. Frente a esto, los proyectos urbanos iconos serán una herramienta muy eficaz, pues permitirán lograr una rápida singularidad y notoriedad en el marketing urbano internacional y con ello, la capacidad de canalizar y atraer nuevos flujos de inversión (Paül i Agustí, 2013).

A pesar de los beneficios que este tipo de proyectos urbanos puede generar en la visión general de los centros urbanos; a nivel local, una débil o inexistente institucionalidad y gobernanza favorecerá el incremento de los desequilibrios e inequidad al interior de las ciudades. Aquellos grupos poblacionales que no disponen de recursos económicos suficientes para satisfacer sus necesidades son relegados, favoreciéndose el incremento de espacios segregados y marginados. Pese a que la planificación estratégica resalta la participación ciudadana y necesaria concertación público-privada, termina siendo el capital económico-político el que definirá las acciones y prioridad proyectos a desarrollar. $Y$ aunque, se creen programas de seguimiento, revisión y ajustes a los planes estratégicos, no siempre se logran los objetivos planteados desde el principio y en lugar de dar respuesta a las necesidades de la población, se agudizan los antiguos y emergen nuevos problemas (Duque, 2014; Precedo et al., 2010).

\section{El arribo de la planificación urbana estratégica en Colombia}

Las principales transformaciones urbanísticas experimentadas por las ciudades colombianas, durante la última década, han sido impulsadas por estrategias gubernamentales en colaboración con agentes privados. Utilizando esta fórmula, los gobiernos locales a través de programas y proyectos de desarrollo municipal y de ordenamiento han intentado plasmar su visión en sus territorios (Arteaga, 2016).

Esta cooperación público-privada a escala local comienza con fuerza a partir de la Política Urbana del Salto Social "Ciudades y Ciudadanía" de 1995 y de la Ley 388 de 1997 de Desarrollo Territorial, con las cuales los municipios obtuvieron un mayor liderazgo y, a su rol de prestador de servicios y ejecutor de obras de infraestructura, se les agregó la labor de "gestión y administración de tales acciones asumiéndolas no como un fin, sino como un medio para impulsar su vocación económica" (Ministerio de Desarrollo Económico, 1996).

Estos cuerpos legales buscaron incentivar la competitividad de los municipios por la atracción de recursos económicos, a través de la generación de distintos instrumentos y directrices, tales como son los "Acuerdos Urbanos de Competitividad" que estaban orientados a favorecer la competencia de los entes territoriales mediante programas regionales de desarrollo científico y tecnológico, planes estratégicos de exportación (e.g. zonas francas, devolución de impuestos), modernización industrial y de infraestructura; "Acuerdos Urbanos de Solidaridad" que abarcaban la constitución de una red de solidaridad social (e.g. inversiones públicas en ámbitos sociales), política de vivienda social urbana (e.g. instrumentos financieros de la reforma urbana), programas de generación de empleo y prestación de servicios públicos; "Acuerdos Urbanos de Sustentabilidad Ambiental" que contemplan la concertación entre instituciones ambientales y administraciones locales y la promoción de tecnologías limpias; “Acuerdos Urbanos para la prevención de desastres" creando una base datos e incorporando las variables de riesgos y desastres en los instrumentos de planificación y ordenamiento territorial; y los "Acuerdos de Desarrollo Urbano Regional", que buscaban el fortalecimiento y la promoción de centros urbanos para la internacionalización (Ministerio de Desarrollo Económico, 1996).

También se crearon los Macroproyectos Urbanos Estratégicos que pretendían impulsar un tratamiento integral de las intervenciones urbanas, las que debían ser de iniciativa local, gestionados públicamente y con una fuerte participación privada. Como características más destacables para categorizar a las iniciativas como un macroproyecto se resaltaban: Gran impacto, propuestas 
que tenían alto potencial estructurales en las ciudades (e.g. viviendas de interés social, proyectos viales y de transporte, renovación urbana, rehabilitación de zonas subnormales); Integralidad, son intersectoriales e interdimensionales; Concentración, al tener una participación activa de los actores urbanos; Interinstitucionales, en ellos participan diferentes niveles de la administración pública; Alcance intra e interubano, afectan la totalidad de los centros urbanos y tienen un impacto sobre otros asentamientos cercanos; y Compromiso local, al otorgar a los municipios la responsabilidad de planear, gerenciar y asignar recursos para la ejecución (Ministerio de Desarrollo Económico, 1996).

Por otro lado, la Ley 388 de 1997 establece mecanismos que permiten a los municipios promover el ordenamiento de su territorio, entendido este último como un conjunto de acciones político-administrativas y de planificación física que les permitan orientar el desarrollo del territorio local de acuerdo con las estrategias de desarrollo socioecómico, en armonía con el medio ambiente y las tradiciones históricas y culturales. Para lograr estos objetivos, el plan de ordenamiento se hace hincapié en que se deberá, entre otras cosas, identificar las ventajas comparativas de los territorios y lograr su mayor competitividad. A su vez, en el artículo № 3 se establecer la función pública del urbanismo, es decir, el ordenamiento del territorio debe posibilitar a los habitantes el acceso a infraestructura, espacios público y servicios básicos; la utilización racional de los usos del suelo en aras del interés común; mejoramiento de la calidad de vida de los habitantes, distribución equitativa de las oportunidades y beneficios del desarrollo; y mejorar la seguridad de los asentamientos humanos ante los riesgos naturales.

A pesar de las intenciones y objetivos trazados los instrumentos mencionados en ambas leyes se han ejecutado de manera fragmentada en las ciudades, favoreciendo a determinados sectores y no a la totalidad del territorio municipal. Así los proyectos urbanos realizados adquieren la forma de enclaves que han favorecido nuevas formas de desigualdad socio-espacial, con ciertos niveles de desregulación o subsidios que alteran la capacidad portante de los lugares (Alzate, 2018).

\section{Metodología}

Para la realización de este trabajo se realizó una búsqueda bibliográfica que permitió la construcción del marco teórico sobre la relevancia de la planificación urbana estratégica y la emergencia de los proyectos urbanos iconos como instrumento para el posicionamiento de las ciudades en el marketing urbano internacional y para la atracción de capitales. Así también se utilizaron referencias bibliográficas para reconstruir los alcances del macroproyecto La Ronda del Sinú. Finalmente, para conocer la percepción en la población sobre los aspectos positivos y negativos sociales, económicos y culturales generadas por la construcción del proyecto urbano se realizaron 15 entrevistas semiestructuradas a habitantes/usuarios que habitan o trabajan en el área de influencia directa del macroproyecto. Dichas entrevistas fueron realizadas entre los meses de mayo y septiembre de 2017 y la información recolectada fue analizada e interpretada siguiendo una metodología cualitativa de análisis de contenido (Bardin, 1986).

\section{El macroproyecto urbano "La Ronda del Sinú" en la ciudad de Montería}

San Jerónimo de Montería se encuentra ubicada en el Departamento de Córdoba, Colombia. Fue fundada el 1 de mayo de 1777 y se encuentra emplazada sobre el valle del río Sinú, la morfología de la ciudad muestra un componente histórico en el que se pueden ver los vestigios de la cultura española, quien impregnó en el territorio monteriano los estilos de plazas, planos urbanos, organización, entre otros, propios del país europeo. De acuerdo con la jerarquía de urbana existente en Colombia, Montería es considerada como una ciudad intermedia y cuenta con una población de 352.388 habitantes (DANE, 2012); históricamente ha sido el principal centro de comercio y prestación de servicios financieros, institucionales, de salud y educación del departamento de Córdoba, destacándose además por el desarrollo de actividades agrícolas y ganaderas de importancia regional y nacional, también cuenta un puerto aéreo de carácter internacional y un terminal de transporte terrestre con amplia cobertura de rutas en el país, aspectos que favorecen el desarrollo de la industria turística. 
El diseño del Parque Lineal La Ronda del Sinú, es una obra urbanística pensada para reintegrar a la ciudad de Montería y a sus habitantes con el río y posibilitar la coexistencia de espacios de conservación ambiental, recreación y actividades económicas de forma ordenada. El objetivo de su construcción y diseño estaba vinculado a la adecuación y rehabilitación del espacio público, que según lo establecido en la Ley 388 de 1997 corresponde a los inmuebles públicos y los elementos arquitectónicos y naturales de los inmuebles privados, destinados a la satisfacción de necesidades urbanas colectivas que superan los límites de los intereses individuales de los habitantes (Figura 1).

Considerando tal definición y por iniciativa del gobierno local en el periodo 2001-2003, se habilitó un concurso público para el diseño y construcción del parque que se localizaría inicialmente en la margen derecha del río Sinú y que a largo plazo se extendería hacia el margen izquierdo del río, área de la ciudad caracterizada por la presencia de asentamientos con bajos niveles de ingreso socioeconómico y en situación de desplazamiento por
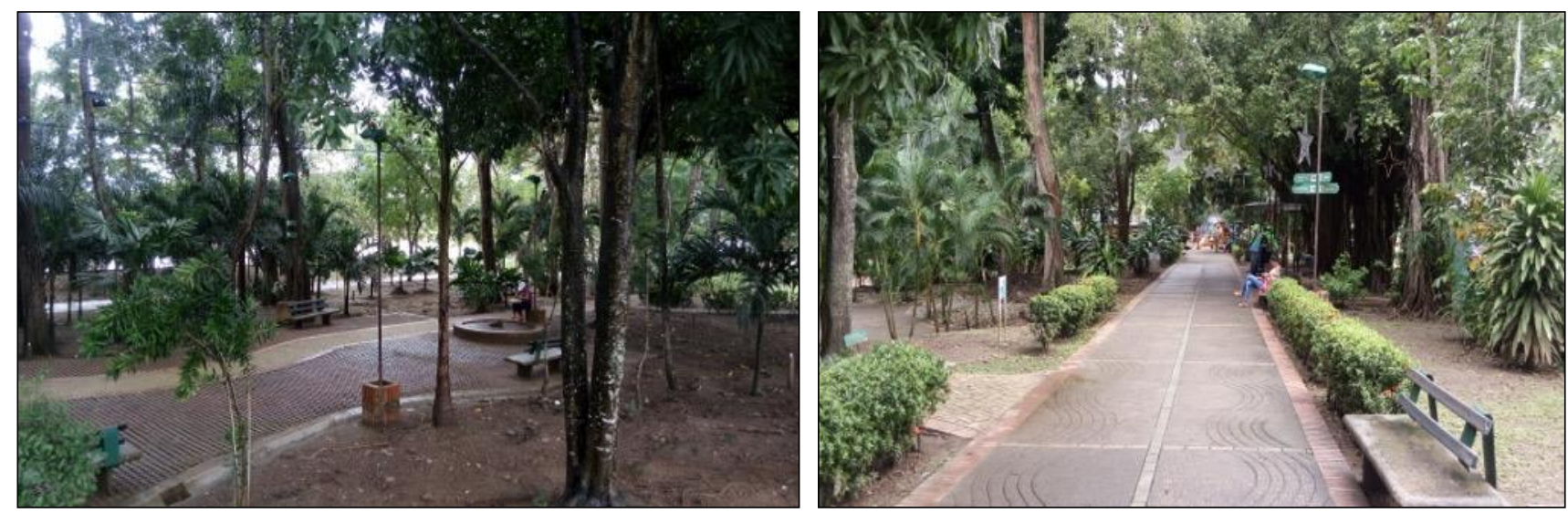

Figura 1. Senderos, vegetación y franja peatonal en la Ronda del Sinú Fuente: Elaboración propia.

causa de la violencia experimentada en el mundo rural. La empresa Parques Nueva Montería fue la responsable de contratar un equipo de arquitectos que partiendo de la base natural existente en la ribera del río diseñaron un parque que conservaría la fauna y flora asociada al lugar, e "invitaría" a los ciudadanos.

El proceso de construcción de la Ronda del Sinú estuvo dividido en varias etapas, dado que fue visionado para su desarrollo a largo plazo. Además de depender de la voluntad política de los gobiernos locales y su interés por continuar el proyecto iniciado por el alcalde Luis Alfredo Jiménez Espitia, la construcción de las etapas proyectadas también fue estimulada por la ciudadanía, la cual expresaba en cada periodo de elección de un nuevo gabinete de gobierno, la importancia de concluir una obra de impacto regional para el aprovechamiento y disfrute del espacio público. De la misma manera, la alianza entre el sector público y la empresa privada facilitó la continuidad del proyecto, logrando una inversión de US\$4.500.000.- en un total de 110.000 metros cuadrados (Alcaldía de Montería, 2011, p. 25). 


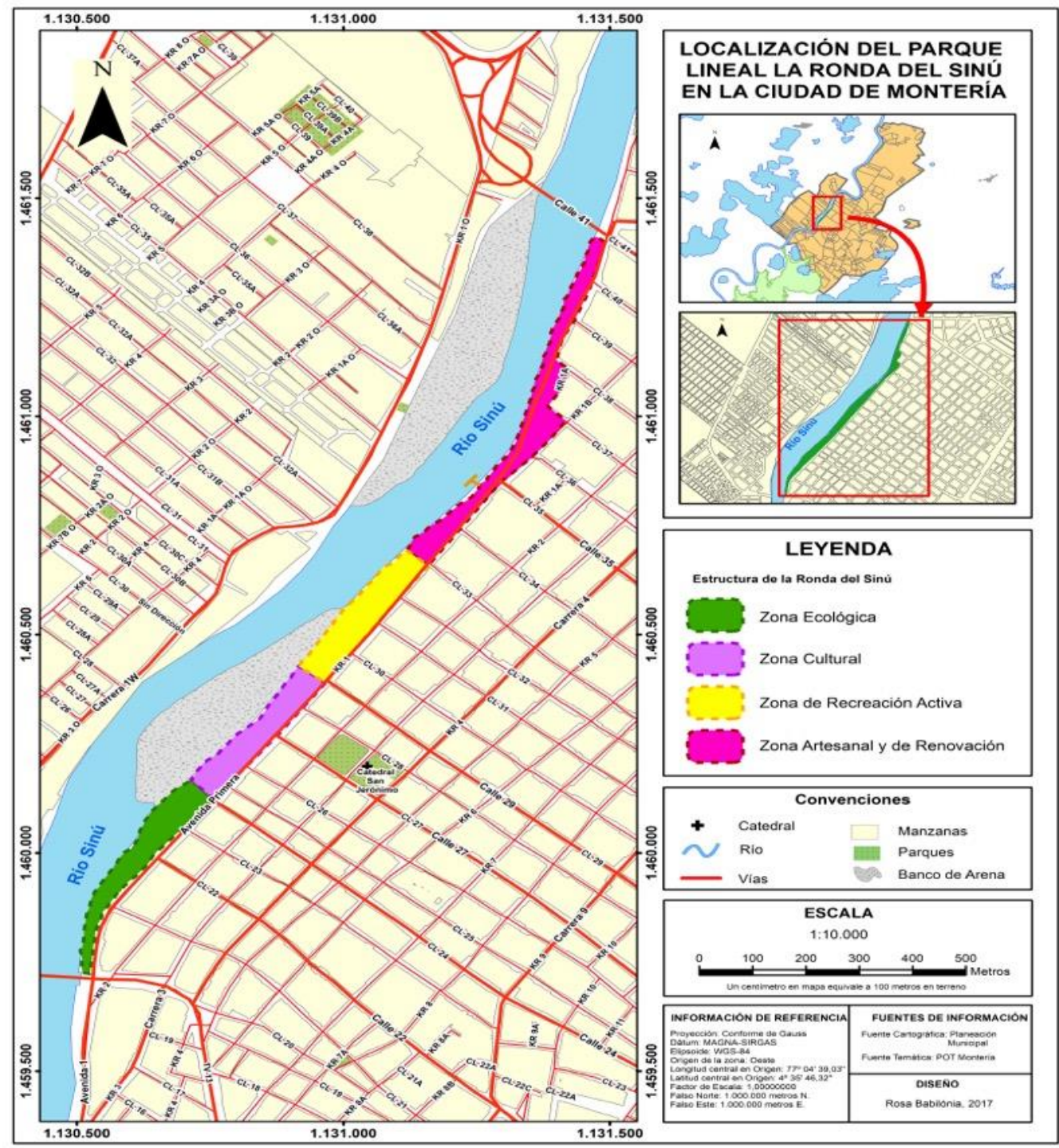

Figura 2. Estructura del Parque Lineal Ronda del Sinú, Montería - Colombia

Fuente: Elaboración propia.

Durante la implementación del proyecto entre el año 2005 y 2010 se construyeron los tramos de la Ronda centro y Ronda norte, la primera se extiende desde la calle 21 a la calle 38 y ocupa un total de $2,5 \mathrm{~km}$ lineales $(63.200$ $\mathrm{m}^{2}$ ) para lo cual fue necesario reubicar la zona de comercio informal existente la cual se caracterizaba por la presencia de vendedores de pescado, verduras y frutas. La segunda fase se construyó entre las calles 56 a la 62 , con un total de $700 \mathrm{~m}$ lineales $\left(17.500 \mathrm{~m}^{2}\right)$, y cuenta con un mirador de $15 \mathrm{~m}$ de altura, escenarios culturales y ciclovías. La última fase, se proyectó hacia el sur de Montería con el propósito de integrar las áreas marginales de la ciudad y mejorar el espacio público; sin embargo, esta etapa no se ha llevado a cabo hasta la actualidad (Figura 2).

\section{Percepción ciudadana sobre el parque lineal "La Ronda del Sinú" en la ciudad de Montería}

La elaboración del proyecto buscaba adecuar el espacio público, creando un lugar para la sociabilización y la vida comunitaria; la conservación de especies vegetales nativas, que permitiera proteger y mantener la 
biodiversidad; y constituir el inicio de una planificación que condujera a la solución de conflictos por el uso del suelo en la ciudad.

La mayoría de los ciudadanos señalan que estos objetivos constituían antiguas demandas de los habitantes cercanos a la ribera del río Sinú y por ello, que fueran abordados por este proyecto era altamente valorado. Por otro lado, los usuarios destacan que, gracias al parque lineal:

la ciudad de Montería cambió notoriamente su imagen. Actualmente, es una ciudad muy visitada, y en especial su ronda, donde se puede apreciar tranquilidad y un ambiente agradable. Ahora es un lugar donde los monterianos pueden realizar actividades al aire libre, practicar deporte, llevar a los niños a las zonas de juegos y descansar un poco del ámbito citadino, ya que años anteriores la ciudad no contaba con un espacio público agradable de esta magnitud (Estudiante, 25 años).

De esta manera, se observa que los ciudadanos reproducen el discurso de la planificación urbana estratégica, en el sentido de considerar que el proyecto lineal constituye un hecho que le permite a Montería posicionarse en el mapa del urbanismo nacional, lo cual "ha significado mucho para nosotros los monterianos, y que si se siguen estas obras en la ciudad [...] Montería va a ser muy atraída por otros habitantes (Estudiante, 25 años).

Junto con destacar el positivo marketing logrado por la ciudad, los usuarios reconocen que estos cambios solo han afectado al "sector norte y centro de la ciudad" y, por tanto, sus beneficios no han favorecido al resto de la urbe. A pesar que los proyectos urbanos, según la Ley de 1997, debían buscar un mejoramiento de la calidad de vida de todos los habitantes y lograr una distribución equitativa de las oportunidades y beneficios, las autoridades locales de Montería han descuidado estos fines, cayendo en:

Hasta cierto punto en una especie de 'populismo urbano', puesto que se preocupan por mostrar el parque, por fotografiarlo y mostrarlo al mundo, pero se olvidan de la gente (Periodista, 35 años).

A nivel de microescala del proyecto urbano, los habitantes reconocen un mayor número de deficiencias, las cuales se originarían en la "escasa participación de los vecinos" en la elaboración del plan, incumpliendo uno de los pilares fundamentales de la planificación urbana estratégica, la participación activa de todos los actores urbanos.

Para los habitantes, estas obras dirigidas más bien a lograr un impacto visual y paisajístico, no consideraron la utilización práctica por parte de los potenciales usuarios. Por ejemplo, algunos entrevistados señalaron que el uso del espacio público destinado para la circulación y movilización de peatones y ciclistas no era continuo a lo largo del parque, generando que el uso de este espacio público sea restringido, ya que:

(...) el parque tiene muchos 'laberintos', obstáculos que dificultan la movilidad; las personas deben tener cuidado por dónde caminan para no caerse o estrellarse contra alguna escalera o muro, esto ha obligado a que la ciclorruta que está dentro del parque sea utilizada por los peatones para su movilidad y desplazamiento, lo cual genera discusiones entre quienes conducen bicicletas y quienes se desplazan caminando (Docente, 35 años).

En este mismo sentido, el exceso de ornamentación del parque ha generado el surgimiento de nuevas problemáticas que se han potenciado gracias a los espacios ocultos o de poca visibilidad que existen. Esto se incrementa en determinados momentos del día, donde:

(...) se pierde de cierta forma la luminosidad, lo que provoca un grado de inseguridad en la zona en horas de la tarde y noche, claro esto es evidente solo en sitios donde las luminarias no son suficiente, por ejemplo la parte baja de la calle $23 .$. esa zona por su lejanía al puesto de vigilancia de la policía es una zona bastante insegura, además se evidencia en horas de la noche como lugares de espera para trabajadoras sexuales o habitantes de la calle (Profesional, 26 años).

(...) [al parque] le hace falta mantenimiento y cuidados en la ciclorutas, porque la construyeron muy cerca al río y con los años el río se ha ido erosionando y uno cada vez es más propenso a tener que andar e la bicicleta a una distancia muy cortita del agua...Me parece que también falta seguridad, porque siendo un parque tan grande hay poca vigilancia de la policía, faltan policías 
auxiliares que siempre den vuelta por donde uno está. En las noches duermen indigentes en el parque y hay gente consumiendo droga. Eso cada vez se hace normal $y$ nadie hace nada (Comerciante, 35 años).

Por otra parte, si bien la elección de intervenir inicialmente el río Sinú va en línea con el planteamiento de la planificación estratégica de limitarse a ciertas áreas temáticas y a partir de ahí, tener visión crítica del entorno en cuanto a la determinación de fortalezas y debilidades de la urbe; los ciudadanos señalan que aunque aumentó la "sensibilización" de los monterianos sobre la importancia de conservar el río Sinú y la relevancia que posee realizar una gestión adecuada de su cuenca, el proyecto no ha logrado terminar con uno de los problemas permanentes de la ciudad, las continuas inundaciones de los barrios como consecuencia del crecimiento del caudal del río, pues:

(...) la creciente de río provoca inundaciones en la ronda, impidiendo el libre disfrute de un espacio público (Dueña de casa, 62 años).

Por último, los habitantes/usuarios destacan que los proyectos urbanos y en particular, nuestro objeto de estudio, no logra desarrollar todo su potencial debido a los problemas de los servicios públicos, particularmente, del gobierno local. La débil institucionalidad y gobernanza favorece el incremento de los desequilibrios e inequidad al interior de Montería. Esto genera que aquellos grupos poblacionales que no disponen de recursos económicos suficientes para satisfacer sus necesidades son relegados, favoreciéndose el incremento de espacios segregados y marginados.

En la orilla del río Sinú, los usos del suelo han cambiado a lo largo de su proceso de ocupación. Su composición ha variado desde la conformación de núcleos informales de comercio de víveres y abarrotes, legumbres, frutas o peces hasta el establecimiento de actividades mineroextractivas (principalmente de arena, grava y arcillas), pasando por actividades de comercio de ropa, calzado y materiales de construcción (ferreterías), entre otros (Serrano, 2015).

La adecuación del parque lineal generó el desplazamiento de varios establecimientos comerciales localizados en esta área, así como la reubicación de más de 270 areneros y vendedores informales de verduras. Los entrevistados consideran que la relocalización del comercio, generó un incremento en los niveles de inseguridad en las áreas abandonadas, debido al hecho de que antes los mismos locatarios procuraban contener a los grupos delictuales, pues su accionar perjudicaba sus actividades comerciales.

Por otra parte, la regulación de los locatarios generó que muchos de ellos -especialmente, los de menor capital económico y cultural- no pudieran cumplir con las exigencias en materia tributaria, laboral y sanitaria que el municipio establecía, situación que les impidió poder continuar con sus antiguas actividades comerciales, aumentando las tasas de desempleo de la población más antigua o bien, incrementando la inestabilidad laboral de quienes no pudieron reconvertirse:

(...) al ser [ahora] una zona turística, hay muchos vendedores ambulantes...el empleo informal en esta zona, se ha acentuado. Hay además habitantes de calle que en ocasiones acosan a turistas y locales por cualquier moneda, acentuando la inseguridad y la percepción del miedo en las poblaciones (Geógrafa, 24 años).

Este beneficio desigual generado por el proyecto que reconocen los habitantes va en sintonía con lo expuesto por Duque (2013) respecto a la sobrevaloración que poseen los proyectos urbanos íconos en el sentido de que debido al interés de planear estrategias para exaltar y destacar el valor de las ciudades y alcanzar suficientes niveles de competitividad o notoriedad para atraer flujos de capital, se han desdibujado los problemas de aquellos grupos poblacionales que no disponen de recursos económicos para satisfacer sus necesidades, marginando y segregando estratos de población que habitan determinados sectores de la ciudad por décadas.

\section{Conclusiones}

De acuerdo con lo planteado por Albrechts (2004) la planificación estratégica crea la visión de un entorno futuro, pero todas las decisiones son tomadas en el presente. Esta diferenciación implica que con el tiempo el proceso de planificación estratégica debe considerar constantes evaluaciones con el objetivo de adaptar, modificar y eliminar los lineamientos y acciones establecidas con el fin de tomar oportunas decisiones en cualquier momento. 
En Colombia, este tipo de planificación fue impulsada a partir de la década de 1990, como una manera de fortalecer la democracia, otorgando mayor poder a los gobiernos locales, quienes debían debe luchar por una ciudad más justa y en la cual los ciudadanos, mediante un diálogo abierto lograran un consenso que pudiera aportar de manera creativa en los procesos de toma de decisiones que afecten sus intereses e inquietudes en diferentes niveles.

En el caso de Montería, el macroproyecto de Parque Lineal Ronda del Sinú buscó ser un ejemplo de planeamiento estratégico urbano, inclusivo y responsable. Sin embargo, incorporó una escasa capacidad de diálogo, centrándose en la expresión de interés material, más que en las necesidades reales de los ciudadanos. De esta manera, a diez años de su construcción, los habitantes/usuarios reconocen que el proyecto ha generado un mayor nivel de conocimiento de Montería a nivel nacional e internacional, pero no ha solucionado una serie de desequilibrios entre lo proyectado en la etapa de diseño del plan estratégico y los resultados obtenidos después de su materialización. La etiqueta de ejemplo de ciudad que logró obtener Montería, no eliminó las condiciones sociales y económicas latentes en la ciudad, tales como el desempleo, la inseguridad y el incremento del costo de vida. Los elementos estructurales de desigualdad en el acceso y la distribución de los recursos se mantuvieron y no ha permitido superar las diferencias en la posición social, habilidades y medios económicos.

En definitiva, a partir de esta revisión se podría cuestionar la utilidad que la planificación estratégica urbana aplicada en Colombia, y en muchos otros países de América Latina, tendría para resolver el problema del desarrollo geográfico desigual. No obstante, consideramos que el problema no es el planeamiento estratégico, pues constituye un enfoque adecuado para asumir los desafíos de la actual era de los cambios globales, donde la movilidad de factores de producción, la diversificación de servicios, los flujos de capital y el intercambio tecnológico requieren de respuestas adecuadas por parte de los países y sus centros urbanos. El problema radicaría, en algo que a estas alturas parece un característica inalienable de nuestro continente, la persistencia de una institucionalidad con fisuras e incompleta y una territorialidad parcial, que facilita que las relaciones de poder, en este caso, la planificación urbana, sea permeada por la fuerza política o económica de intereses privados y por tanto, dependa de momentos coyunturales de confrontación entre actores estatales y privados, y los objetivos de la planificación urbana no 


\section{Referencias}

Abbott, C. (2006). Urban History for Planners. Journal of Planning History, 5 (4), 301-313. https://doi.org/10.1177/1538513206293714

Albrechts, L. (2004). Strategic (spatial) planning reexamined. Environment and Planning B: Planning and Design, 31(5), 743-758. https://doi.org/10.1068/b3065

Alcaldía de Montería (2011). Plan Maestro de Cambio Climático Montería Ciudad Verde 2019. Montería, Colombia.

Alzate, A. (2018). Del Urbanismo desarrollista al neoliberal. Estado y producción de espacio urbano en Medellín (1947-2014) (Tesis de Doctorado). Pontificia Universidad Católica de Chile, Santiago, Chile.

Arteaga, M. (2016). Imaginarios urbanos y construcción colectiva del espacio público en la ciudad de Montería (Tesis de pregrado). Universidad de Córdoba. Montería, Colombia.

Bardin, L. (1986). El análisis de contenido. Madrid: Akal.

Brahm, A. (2005). Autopistas urbanas: Santiago, Chile. $A R Q \quad$ (Santiago), (60), 28-39. https://dx.doi.org/10.4067/S071769962005006000005

Brand, P. (1995). Ecologism and Urban Space: Nature, urbanization and city planning in Medellin, Colombia. Planning Practice and Research 10(1) 55-66. http://dx.doi.org/10.1080/02697459550036829

Congreso de Colombia. Ley 388 de 1997. Diario Oficial, № 43.091, 24 de julio de 1997.

De Mattos, C. (2010). Globalización y metamorfosis urbana en América Latina. Quito: OLACCHI-Quito Distrito Metropolitano.

Departamento Administrativo Nacional de Estadísticas (DANE). (2012). Estadísticas de Población. Bogotá, Colombia.

Duque, I. (2011). Bogotá: entre la identidad y el marketing urbano. Cuadernos de Geografía, Revista Colombiana de Geografía 20, 29-45. https://doi.org/10.15446/rcdg.v20n1.23065
Duque, I. (2013). Trayectoria y perspectivas del planeamiento estratégico en las ciudades latinoamericanas. En I. Duque (Eds.), Historiografía y planificación urbana en América Latina. Bogotá: Universidad Nacional de Colombia.

Duque, I. (2014). Políticas públicas, urbanismo y fronteras invisibles. Las disputas por el control espacial en Medellín. Trabajo presentado en el XIII Coloquio Internacional de Geocrítica El control del espacio y los espacios de control. Barcelona, España.

Echabarría, C. y Aguado, I. (2003). La planificación urbana sostenible. Zainak, 24, 643-660.

El-Khoury, R. \& Robbins, E. (Eds.) (2013). Shaping the City: Studies in History, Theory and Urban Design. Abingdon, Oxon: Routledge.

Fainstein, S. (1999). Can we make the cities we want? En S. Body-Gendrot y R. Beauregard (Eds.), The Urban Moment (pp. 249-272). Thousand Oaks: Sage.

Figueroa, O. (2005). Transporte urbano y globalización: Políticas y efectos en América Latina. EURE, 31 (94), 41-53. https://doi.org/10.4067/s025071612005009400003

Gunder, M. (2010). Planning as the ideology of (neoliberal) space. Planning Theory 9, 298-314. https://doi.org/10.1177/1473095210368878

Martínez, A. (2003). Sistema de transporte urbano masivo de pasajeros de Bogotá Transmilenio. Urbano, 6 (7), 31-37.

Mawromatis, C. (2013). Tensiones y convergencia: El diseño urbano contemporáneo como alternativa a la ciudad dispersa y difusa. Revista INVI, 28 (79), 125-163. https://doi.org/10.4067/s0718-83582013000300005

Ministerio de Desarrollo Económico (1996). La Política Urbana del Salto Social. Ciudades y Ciudadanía. Bogotá, Colombia.

Paül i Agustí, D. (2013). Las políticas culturales y sus repercusiones en la imagen de la ciudad. Scripta NovaRevista Electrónica de Geografía y Ciencias Sociales, 17 (432), 1-13.

Pradilla, E. (2009). Las políticas y planeación urbana en el neoliberalismo. En P. Brand (Eds.). La Ciudad 
Latinoamericana en el Siglo XXI: Globalización, neoliberalismo, planeación. (pp. 287- 308). Medellín: Universidad Nacional de Colombia.

Precedo, A., Orosa, J., Míguez, A. (2010). De la planificación estratégica al marketing urbano: hacia la ciudad inmaterial. EURE, 36 (108), 5-27. https://doi.org/10.4067/s0250-71612010000200001

Roitman, S. (2008). Planificación urbana y actores sociales intervinientes: el desarrollo de urbanizaciones cerradas. Trabajo presentado en el $\mathrm{X}$ Coloquio Internacional de Geocrítica: Diez Años de Cambios en el Mundo, en la Geografía y en las Ciencias Sociales, 1999-2008. Barcelona, España.
Serrano, N. (2015). Reubicarán de nuevo a vendedores de verduras del centro de Montería. El Universal.

Sierra, J. (2016). Marketing urbano, forma de gobierno neoliberal en la ciudad de Medellín. Iconofacto, 12 (19),124-153. https://doi.org/10.18566/iconofact.v12.n19.a05

Steinberg, F. (2001). Planificación estratégica urbana en América Latina: experiencias de Construcción y Gestión del Futuro. La Paz, Bolivia: IHS SINPA PAPERS,

Tamayo, D. (2008). Hacia una planificación urbana sistémica: una experiencia universitaria de aprendizaje y aplicación de nuevos instrumentos técnicos en la planificación urbana tradicional. Espacio y Desarrollo, $20,69-86$. 